OPEN ACCESS

Edited by:

Norberto Perico,

Istituto di Ricerche Farmacologiche Mario Negri (IRCCS), Italy

Reviewed by:

Jinghong Zhao,

Xinqiao Hospital, China

Raman Kumar,

Guru Nanak Dev Engineering College,

India

Vincenzo Montinaro, Azienda Ospedaliero Universitaria Consorziale Policlinico di Bari, Italy

*Correspondence: Yongli Zhan zhanyongli88@sina.com

Specialty section: This article was submitted to Renal Pharmacology, a section of the journal Frontiers in Pharmacology

Received: 08 September 2021 Accepted: 16 November 2021 Published: 03 December 2021

Citation:

Liu T, Yang L, Mao H, Ma F, Wang Y and Zhan Y (2021) Knowledge Domain and Emerging Trends in Podocyte Injury Research From 1994 to 2021: A Bibliometric and Visualized Analysis. Front. Pharmacol. 12:772386. doi: 10.3389/fphar.2021.772386

\section{Knowledge Domain and Emerging Trends in Podocyte Injury Research From 1994 to 2021: A Bibliometric and Visualized Analysis}

\author{
Tongtong Liu, Liping Yang, Huimin Mao, Fang Ma, Yuyang Wang and Yongli Zhan* \\ Guang'anmen Hospital, China Academy of Chinese Medical Sciences, Beijing, China
}

Background: Podocyte injury has a direct causal relationship with proteinuria and glomerulosclerosis and, on a chronic level, can lead to irreversible disease progression. Podocyte injury plays a critically decisive role in the development of proteinuric kidney disease. In recent years, the research on podocyte injury has developed rapidly all over the world. However, no report has summarized the field of podocyte injury as a whole to date. Using bibliometric analysis, this study aimed to evaluate the current state of worldwide podocyte injury research in the last 30 years and identify important achievements, primary research fields, and emerging trends.

Methods: Publications related to podocyte injury were retrieved from Web of Science Core Collection. HistCite, VOSviewer, CiteSpace, and the Bibliometrix Package were used for bibliometric analysis and visualization, including the analysis of the overall distribution of annual outputs, leading countries, active institutions and authors, core journals, co-cited references, and keywords. Total global citation score and total local citation score were used to assess the quality and impact of publications.

Results: A total of 2,669 publications related to podocyte injury were identified. Publications related to podocyte injury tended to increase continuously. A total of 10,328 authors from 2,171 institutions in 69 countries published studies related to podocyte injury. China (39.46\%) was the most prolific country, and the number of citations of studies in the United States (cited 36,896 times) ranked first. Moin A Saleem, John Cijiang He, and Zhihong Liu were the top three contributing authors, and Journal of the American Society of Nephrology and Kidney International were the most popular journals in the field. "Diabetic nephropathy" is the primary focus area of podocyte injury research, and "autophagy," "microRNA," and "inflammation" were the top keywords of emerging research hotspots, and traditional Chinese medicine monomer may be a neglected research gap.

Conclusion: Our research found that global publications on podocyte injury have increased dramatically. Diabetic nephropathy is the main research field of podocyte injury, whereas autophagy, microRNA, and inflammation are the top topics getting current attention from scholars and which may become the next focus in podocyte injury research. 


\section{INTRODUCTION}

Podocytes are highly differentiated epithelial cells attached to the surface of the glomerular basement membrane. They play a prominent role in maintaining the integrity of the glomerular filtration barrier. Podocyte injury and loss are closely related to the development of proteinuria and glomerulosclerosis. In 1994, Kretzler $\mathrm{M}$ et al. first attributed glomerulosclerosis to podocyte depletion in a rat model (Kretzler et al., 1994). An important study conducted by Pagtalunan ME et al. found that podocyte loss was related to albuminuria and the deterioration of renal function in Pima Indians with type II diabetes (Pagtalunan et al., 1997). Podocyte depletion is an important morphologic characteristic in the pathogenesis of diabetic kidney disease (DKD) and focal segmental glomerulonephritis (FSGS). Many studies have shown that podocyte injury occur in early-stage DKD (Coimbra et al., 2000) and FSGS (Alachkar et al., 2013), and uncontrollable persistent podocyte injury will lead to glomerulosclerosis and disease progression (Wharram et al., 2005; Makino et al., 2021). Therefore, research focused on preventing podocyte injury or promoting podocyte repair has been the focus and hotspot in this field.

Podocyte injury manifests as hypertrophy, foot process effacement, autophagy, mesenchymal transition, detachment, and apoptosis (Zhou L. et al., 2019). Stress factors that mediate the injury process include mechanical stress (glomerular hypertension or hyperfiltration), oxidative stress, and immune (inflammatory) stress (Nagata, 2016). Once the podocytes are injured, the structure of actin cytoskeleton changes supervenes such that damage to the renal filtration barrier progresses until proteinuria, and glomerular disease occurs (Schell and Huber, 2017). On the other hand, podocyte injury inhibits the synthesis of vascular endothelial growth factor, which in turn affects vascular compartment stabilization and further interrupts the crosstalk between podocytes and vascular compartments, and ultimately leading to the development of glomerulosclerosis. Notably, the proliferation of podocytes is very limited. Although studies have found that podocytes have a certain reserve capacity in infancy, this reserve is rather small ( $<10 \%$ of all podocytes), and becomes depleted with age (Berger et al., 2014; Eng et al., 2015). Mild podocyte injury may be reversible, however, until a certain threshold in podocyte loss is reached ( $>40 \%$ podocyte depletion), and inevitable renal failure occurs (Wharram et al., 2005). Hence, research on podocyte injury is a very promising field, and conducting an analysis of the current status, focus areas, and emerging trends in the field of podocyte injury will yield significant findings.

Bibliometrics is a convenient new approach to the qualitative and quantitative analyses of publications (Chen, 2004; 2017). Using this method, researchers can quickly dig deep into the thematic evolution, main research fields, and new research directions in a certain research field (Chen and Song, 2019).
Bibliometrics has become widely used in many disciplines as an auxiliary research method (Wang et al., 2021). However, bibliometric studies on podocyte injury remains lacking.

In this study, we used bibliometric methods to analyze the publications on podocyte injury and systematically evaluate the research status, current research focus, emerging research trends of podocyte injury in the past three decades, highlighting landmark achievements, and pointing out directions for future research.

\section{MATERIALS AND METHODS}

\section{Data Source and Search Strategy}

We conducted a literature search on the Web of Science Core Collection (WoSCC) on podocyte injury in the past 30 years (from 1990 to 2021). The search formula was as follows: TS = ("podocyte pyroptosis" OR "podocyte apoptosis" OR "podocytopathy" OR "podocytopathies" OR "podocyte injury" OR "podocyte damage", and OR "podocyte dysfunction"). The article language was set to English. In order to avoid deviations from data updates, all the above operations were performed within 1 day, and on July 4, 2021.

\section{Eligibility Criteria and Data Collection}

The document types included in the study were only articles and reviews. Meeting abstracts, editorial materials, and proceedings papers, among others, and were excluded. Duplicate studies were also removed artificially. All the information, including the number of papers and citations, titles, authors, affiliations, countries, keywords, journal, publication year, and references, were collected for bibliometric analysis.

\section{Statistical Analysis}

In this study, HistCite (version 12.03.17), VOSviewer (version 1.6.16), CiteSpace (version 5.7.R5), and the Bibliometrix 4.1.0 Package (https://www.bibliometrix.org) based on the R language were used to perform the bibliometric analysis.

HistCite (Garfield et al., 2006) was used to calculate the total number of publication records, total global citation score (TGCS), and total local citation score (TLCS) for each publication year, active countries, top institutions, core journals, and authors. More importantly, it was used to identify the representative citation paths of important references.

VOSviewer (van Eck and Waltman, 2010) was used to visualize complex co-citation networks, such as the cooperation and time trends among countries, institutions, and individuals. The size of the nodes represents the number of publications; the thickness of the line represents the strength of the link; and the colors of the nodes represent different clusters or times.

CiteSpace was used to aid visual analysis of the knowledge domain and emerging trends (Chen, 2004), including cluster analysis, dual-map overlay of citations, timeline or time zone 
A
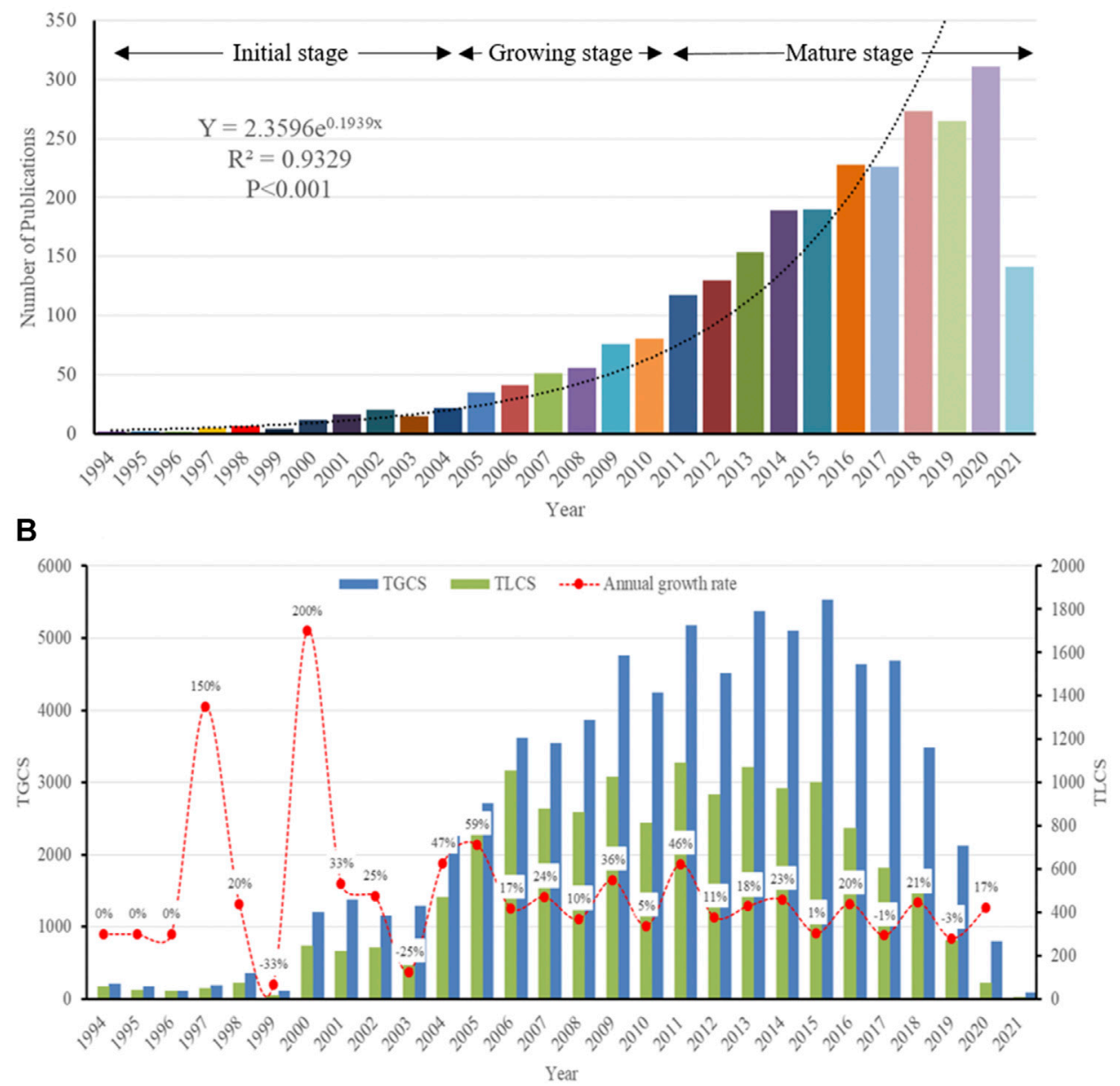

FIGURE 1 | Overall Distribution of publication outputs on podocyte injury (A) Global annual output trends; (B) TGCS, TLCS and growth rate in annual publications.

views, references, and keywords citation bursts (Chen and Leydesdorff, 2014; Chen and Song, 2019). Cluster analysis can classify references and keywords and identify important research areas on podocyte injury. The modularity $\mathrm{Q}$ and mean silhouette are two important evaluation indicators in cluster analysis. Q > 0.3 indicates that the clustering structure is significant enough. Mean silhouette $>0.5$ indicates that the clustering results are convincing. The bursts of keywords and references are often used to detect new research trends in the field.

The Bibliometrix Package is an established tool based on the $\mathrm{R}$ language that is used for bibliometric analysis (Aria and Cuccurullo, 2017). We conducted a thematic evolution analysis using the Bibliometrix Package to categorize the changes in podocyte injury research into different periods.

\section{RESULTS}

\section{Overall Distribution}

A total of 2,669 publications related to podocyte injury were retrieved from WoSCC, including 2,311 articles, and 358 reviews
(Supplementary Table S1; Supplementary Figure S1). Curve fitting analysis showed that the annual number of publications on podocyte injury has undergone an overall increasing trend since $1994\left(\mathrm{R}^{2}=0.9329 ; p<0.001\right)$. We artificially divide this period into three stages according to the annual output and growth rate: Initial stage (1994-2004), growing stage (2005-2010), and mature stage (2011-2021). In the initial stage, the total number of publications on podocyte injury was less than 20 per year, and in 1994-1996, only two articles were published in each year, and the only two articles in 1994 were written by Pascua $M$ and Kretzler M, who investigated podocyte injury earlier than researchers in other parts of the world. Pascual et al. (1994) found that complement receptor 1 (CR1) can be used as a marker for podocyte injury (cited 68 times). Over the same period, Kretzler et al. (1994) proposed for the first time that in the uninephrectomized-desoxycorticosterone hypertensive rat model, and glomerulosclerosis may be due to podocyte injury (cited 143 times). In the growing stage, the number of total publications on podocyte injury was less than 100 per year, but increased at an average rate of 9.6 articles per year, and with an average annual growth rate of $25.23 \%$. In the mature stage, the 


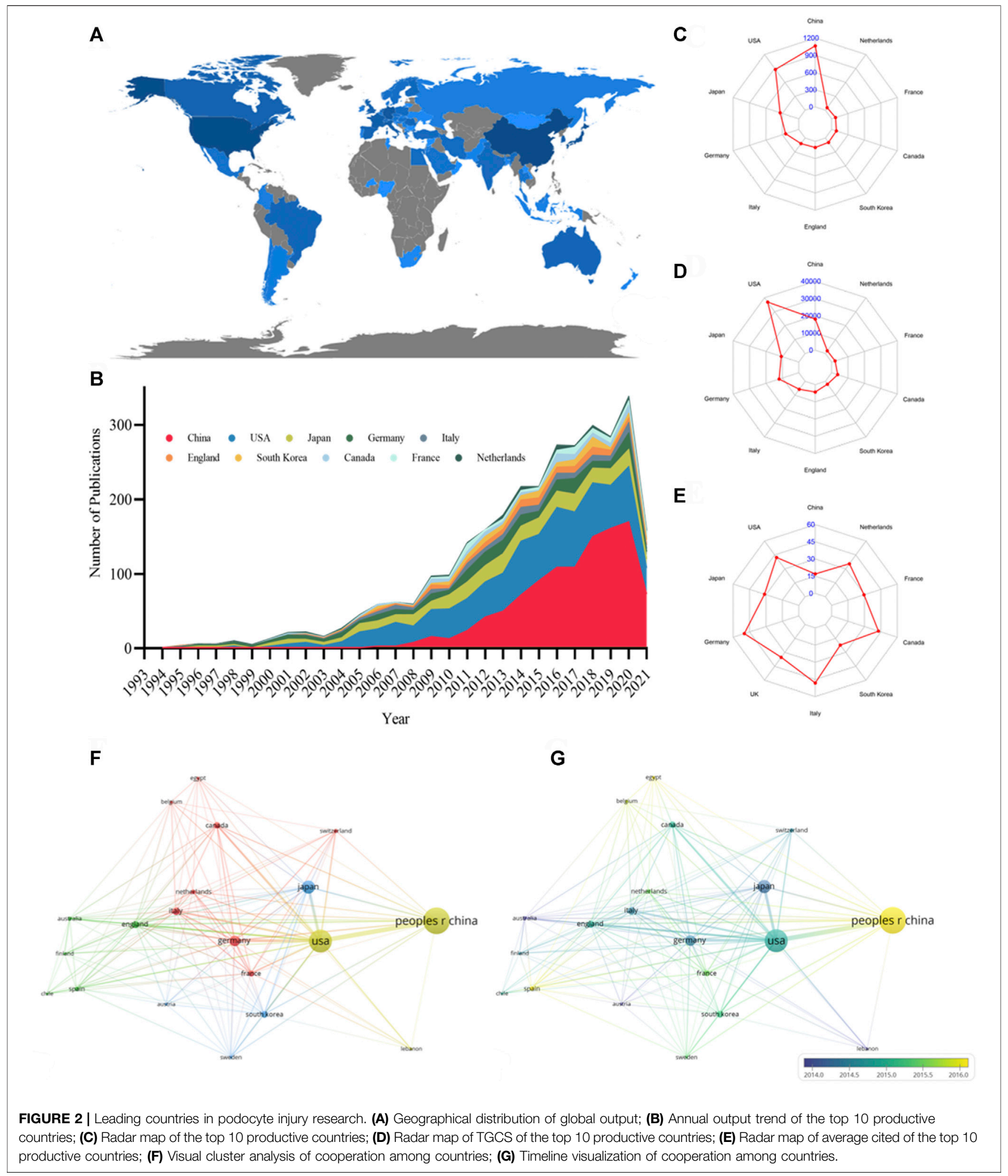

total number of publications on podocyte injury was more than 100 per year and increased at an average rate of 23.1 articles per year, with an average annual growth rate of $14.76 \%$ (Figure 1A).
The highest number of articles was published in $2020(n=311)$, which was more than the total number of articles published in the previous 15 years $(1994-2008, n=289)$. 
TABLE 1 | The top 10 productive countries concerning podocyte injury research.

\begin{tabular}{|c|c|c|c|c|c|c|}
\hline Rank & Country & $\begin{array}{c}\text { Publications } \mathbf{n} \\
\text { (\%) }\end{array}$ & LCS & TGCS & Average citation & Centrality \\
\hline 1 & China & 1,065 (39.90\%) & 3,739 & 18,077 & 16.97 & 0.03 \\
\hline 2 & United States & 878 (32.67\%) & 7,651 & 36,896 & 42.31 & 0.54 \\
\hline 3 & Japan & 342 (12.78\%) & 2,453 & 10,689 & 31.35 & 0.08 \\
\hline 4 & Germany & 242 (9.03\%) & 2,399 & 12,008 & 49.83 & 0.29 \\
\hline 5 & Italy & 120 (4.57\%) & 1,030 & 5,714 & 35.28 & 0.16 \\
\hline 6 & England & 107 (4.46\%) & 648 & 4,304 & 48.02 & 0.09 \\
\hline 7 & South Korea & 93 (3.48\%) & 393 & 2056 & 22.11 & 0.03 \\
\hline 8 & Canada & 86 (3.22\%) & 627 & 3,680 & 42.79 & 0.06 \\
\hline 9 & France & 72 (2.66\%) & 338 & 2,100 & 29.58 & 0.16 \\
\hline 10 & Netherlands & 54 (2.02\%) & 425 & 1919 & 35.54 & 0.06 \\
\hline
\end{tabular}

TABLE 2 | The top 10 productive institutions concerning podocyte injury research.

\begin{tabular}{|c|c|c|c|c|c|}
\hline Institution & Country & Publication counts & TGCS & TLCS & Average citation \\
\hline Southern Medical University & China & 68 & 1951 & 371 & 28.69 \\
\hline Nanjing Medical University & China & 64 & 1,426 & 373 & 22.28 \\
\hline University of Bristol & United Kingdom & 62 & 2067 & 328 & 33.34 \\
\hline University of Michigan & United States & 62 & 3,280 & 739 & 52.90 \\
\hline Nanjing University & China & 60 & 1,645 & 352 & 27.42 \\
\hline University of Washington & United States & 57 & 3,986 & 1,051 & 69.93 \\
\hline Shanghai Jiao Tong University & China & 56 & 1,177 & 193 & 21.02 \\
\hline Fudan University & China & 53 & 903 & 191 & 17.04 \\
\hline Shandong University & China & 49 & 903 & 197 & 18.43 \\
\hline Icahn School of Medicine at Mount Sinai & United States & 47 & 973 & 230 & 20.70 \\
\hline
\end{tabular}

So far, these articles have been cited 72,836 times, with an average of 27.29 times per article. Since the field of research was then still immature, the TGCS and TLCS of articles published in the initial stage were low. However, the TGCS increased year by year from 2005 to 2008. Since 2009, the TGCS has been relatively stable, indicating that the research on podocyte injury has entered a relatively mature stage (Figure 1B).

\section{Leading Countries}

From 1994 to 2021, 69 countries published research articles on podocyte injury. The global article productivity is presented in Figure 2A. The top 10 countries with the highest number of publications have generated about $84.57 \%$ of the articles in the world (Figure 2B; Table 1). China showed the highest output, publishing a total of $1,065(39.46 \%)$ articles related to podocyte injury, followed by the United States ( $n=872 ; 32.31 \%)$, and Japan ( $n=341 ; 12.63 \%$ ) (Figure 2C). The most cited country for published articles is the United States (cited 36,896 times), followed by China (cited 18,077 times), and Germany (cited 12,008 times) (Figure 2D). In addition, German publications have the highest average number of citations (average cited 49.83 times), followed by Italy (average cited 48.02 times), and Canada (average cited 42.79 times) (Figure 2E). The visualized international collaboration network showed that the cooperation between countries is relatively close. The United States and China showed the closest cooperation, and the United States has been in cooperation with almost all the other countries (Figure 2F). Since 1994, research in this field has increased in Germany, Italy, and the United Kingdom, whereas the research on podocyte injury in China, Spain, and Egypt has increased since 2005 (Figure 2G).

\section{Active Institutes and Authors}

A total of 10,328 authors from 2,172 institutions have published articles on podocyte injury. The top 10 institutions with the highest output in podocyte injury research are shown in Table 2. Southern Medical University in China $(n=68)$ was the leading institution in terms of output, followed by Nanjing Medical University in China $(n=64)$, the University of Bristol in the United Kingdom $(n=62)$, and the University of Michigan in the United States $(n=62)$. The TGCS of the University of Washington in the United States (cited 3,986 times) was the highest, followed by the University of Michigan (cited 3,280 times), and the University of Bristol (cited 2067 times). Cooperation among institutions was relatively close and was divided into four institutional clusters. The cooperation groups led by the University of Michigan showed the closest cooperation with other institutions (Figure 3A).

The top three most productive authors were Moin A Saleem of the University of Bristol (published 50 articles), John Cijiang He of the Icahn School of Medicine at Mount Sinai (published 43 articles), and Zhihong Liu of the Medical School of Nanjing University (published 40 articles) (Table 3). Kretzler M is an early researcher on podocyte injury. He published his first achievement in this field in 1994. So far, he has been engaged in research in this field for nearly 30 years (1994-2020) (Figure 3B). Youhua Liu of 


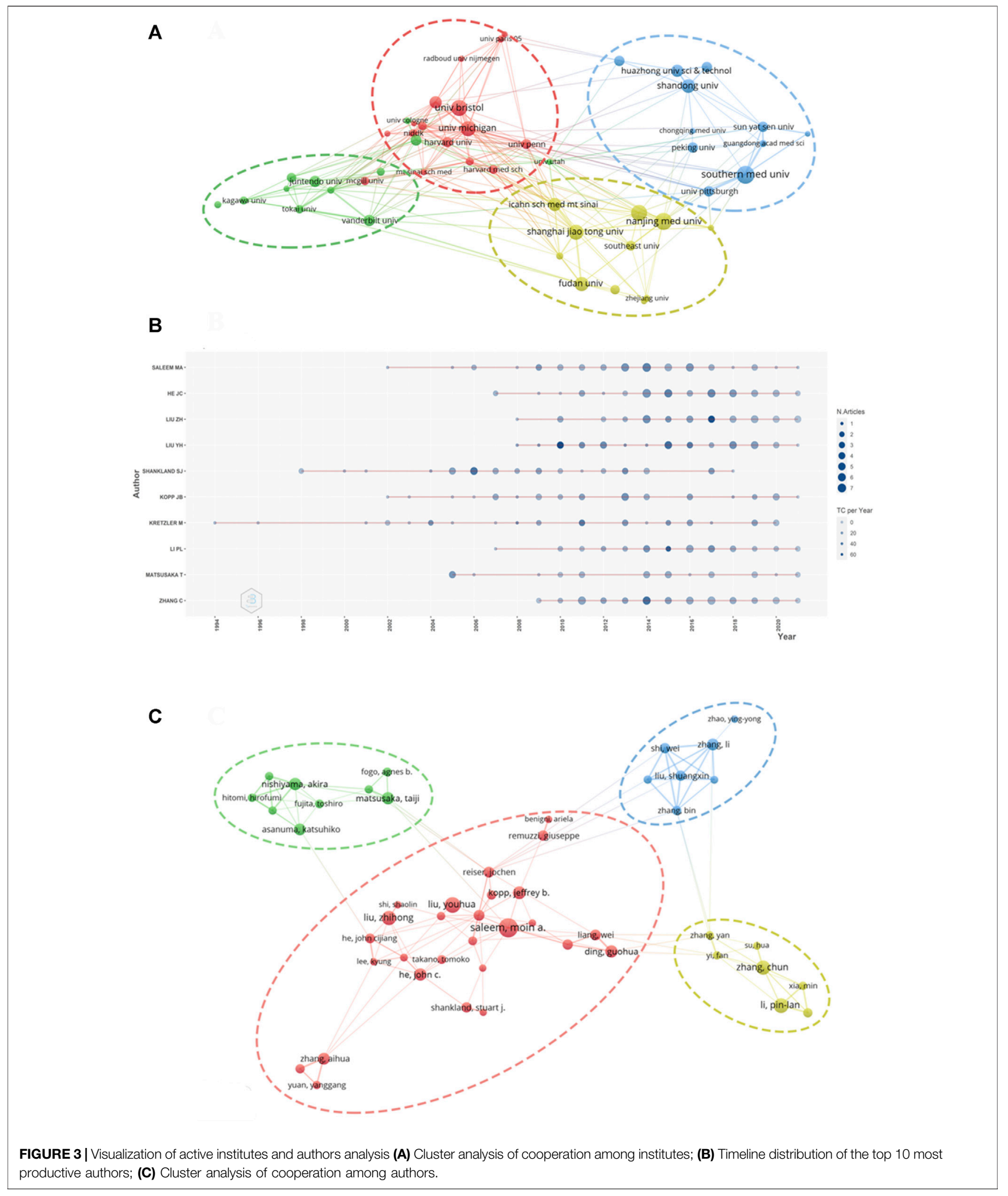


TABLE 3 | The top 10 most productive authors in podocyte injury research.

\begin{tabular}{|c|c|c|c|c|c|c|c|}
\hline Rank & Name & Country & Institution & Counts & TLCS & TGCS & H-index \\
\hline 1 & Moin A Saleem & United Kingdom & Univ Bristol & 50 & 280 & 1792 & 24 \\
\hline 2 & John Cijiang He & United States & Icahn Sch Med Mt Sinai & 43 & 309 & 1,212 & 20 \\
\hline 3 & Zhihong Liu & China & Nanjing Med Univ & 40 & 317 & 1,365 & 18 \\
\hline 4 & Youhua Liu & China & Southern Med Univ & 35 & 688 & 3,120 & 25 \\
\hline 5 & Stuart J Shankland & United States & Univ Washington & 37 & 872 & 2,730 & 29 \\
\hline 6 & Jeffrey B Kopp & United States & $\mathrm{NIH}$ & 35 & 270 & 1,417 & 22 \\
\hline 7 & Pin-Lan Li & United States & Virginia Commonwealth Univ & 32 & 279 & 1,330 & 19 \\
\hline 8 & Matthias Kretzler & United States & Univ Michigan & 32 & 563 & 2,613 & 24 \\
\hline 9 & Taiji Matsusaka & Japan & Tokai Univ & 29 & 282 & 927 & 15 \\
\hline 10 & Chun Zhang & China & Huazhong Univ Sci \& Technol & 29 & 286 & 1,004 & 16 \\
\hline
\end{tabular}

TABLE 4 | The top 10 core journals on podocyte injury research.

\begin{tabular}{|c|c|c|c|c|c|c|}
\hline Rank & Journal & Counts & TLCS & TGCS & IF (2020) & H-index \\
\hline 1 & Journal of the American Society of Nephrology & 159 & 2,110 & 10,305 & 10.121 & 60 \\
\hline 2 & Kidney International & 148 & 2072 & 8,895 & 10.612 & 55 \\
\hline 3 & American Journal of Physiology-Renal Physiology & 142 & 1,027 & 4,476 & 3.377 & 38 \\
\hline 4 & Plos One & 94 & 0 & 2,182 & 3.240 & 26 \\
\hline 5 & Nephrology Dialysis Transplantation & 75 & 525 & 2,440 & 5.992 & 30 \\
\hline 6 & Scientific Reports & 67 & 0 & 944 & 4.379 & 18 \\
\hline 7 & Biochemical and Biophysical Research Communications & 49 & 176 & 560 & 3.575 & 15 \\
\hline 8 & American Journal of Pathology & 36 & 553 & 1752 & 4.307 & 24 \\
\hline 9 & Journal of Biological Chemistry & 35 & 462 & 1857 & 5.157 & 24 \\
\hline 10 & Molecular Medicine Reports & 31 & 62 & 291 & 2.952 & 10 \\
\hline
\end{tabular}

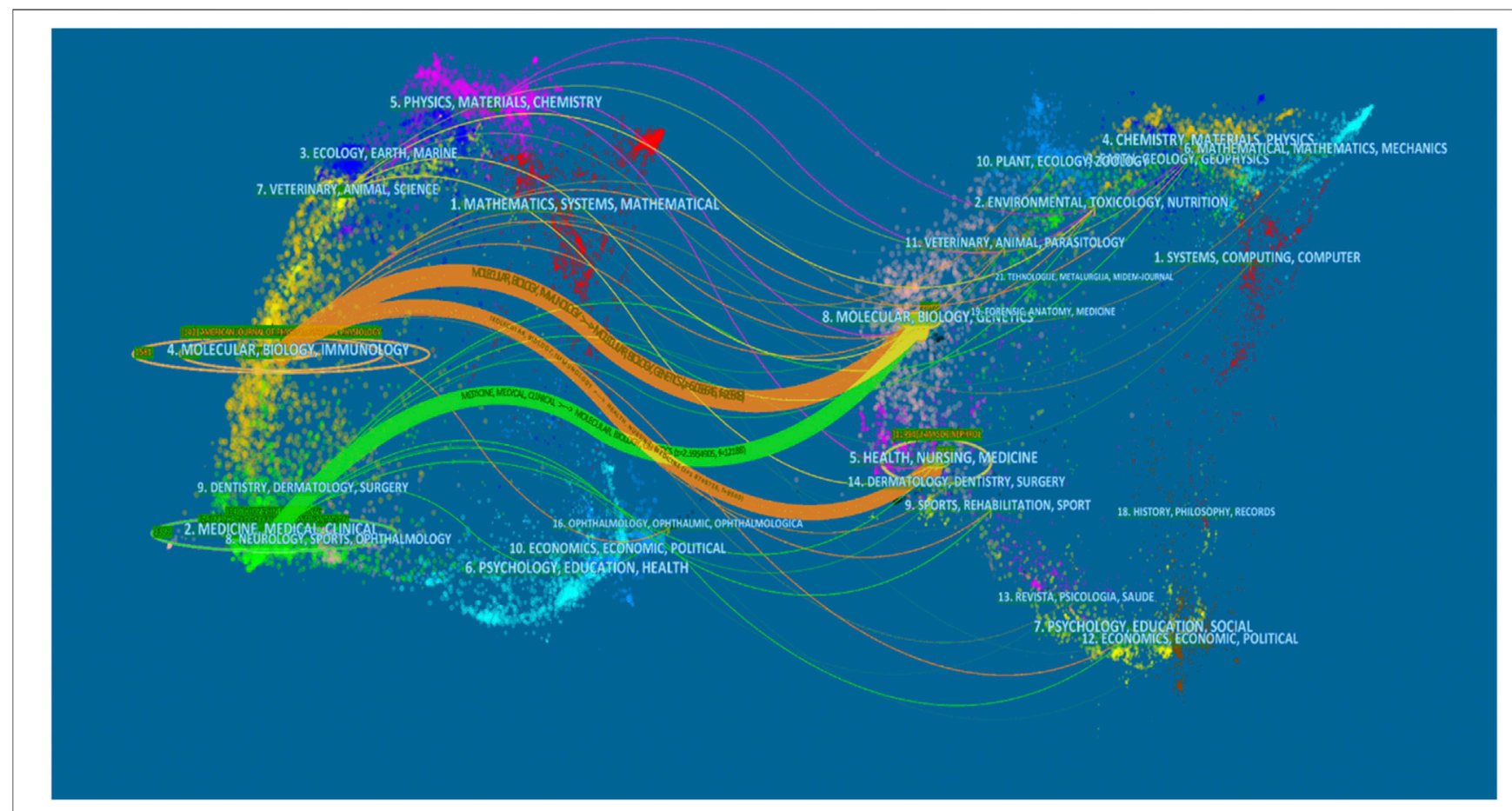

FIGURE 4 | The dual-map overlay of articles citing on podocyte injury research. (The left side were the citing journal, the right side were the cited journal, and the line path represents the citation relationship). 
TABLE 5 | The top 10 literatures with the highest number of citations.

\begin{tabular}{|c|c|c|c|c|c|c|c|c|}
\hline Rank & $\begin{array}{c}\text { First } \\
\text { author }\end{array}$ & Journal & Year & IF (2020) & Category & Cluster & TGCS & TLCS \\
\hline 1 & Susztak K & Diabetes & 2006 & 9.461 & Pathophysiology & \#2 & 767 & 268 \\
\hline 2 & Shankland SJ & Kidney International & 2006 & 10.612 & Pathophysiology & \#5 & 589 & 249 \\
\hline 3 & Liu YH & Journal of the American Society of Nephrology & 2010 & 10.121 & Pathophysiology & \#3 & 580 & 53 \\
\hline 4 & Wiggins RC & Kidney International & 2007 & 10.612 & Pathophysiology & $\# 5$ & 494 & 191 \\
\hline 5 & Eddy AA & Lancet & 2003 & 79.321 & Pathophysiology & \#5 & 460 & 19 \\
\hline 6 & Wolf G & Diabetes & 2005 & 9.461 & Treatment & \#2 & 454 & 185 \\
\hline 7 & Sharma K & Journal of Clinical Investigation & 2008 & 14.808 & Treatment & \#3 & 446 & 44 \\
\hline 8 & Reiser J & Journal of Clinical Investigation & 2004 & 14.808 & Injury mechanism & \#5 & 384 & 96 \\
\hline 9 & Wei C & Nature Medicine & 2008 & 53.44 & Injury mechanism & \#0 & 375 & 82 \\
\hline 10 & Ronconi E & Journal of the American Society of Nephrology & 2009 & 10.121 & Treatment & $\# 5$ & 358 & 55 \\
\hline 11 & Inoki K & Journal of Clinical Investigation & 2011 & 14.808 & Injury mechanism & $\# 1$ & 355 & 87 \\
\hline 12 & Abais JM & Antioxidants \& Redox Signaling & 2015 & 8.401 & Injury mechanism & $\# 5$ & 347 & 8 \\
\hline 13 & Coimbra TM & Kidney International & 2000 & 10.612 & Injury mechanism & $\# 4$ & 301 & 50 \\
\hline 14 & Trimarchi H & Kidney International & 2017 & 10.612 & other & \#8 & 289 & 4 \\
\hline 15 & Coughlan MT & Journal of The American Society of Nephrology & 2009 & 10.121 & Injury mechanism & $\# 4$ & 287 & 3 \\
\hline
\end{tabular}

TABLE 6 | The top 6 clusters of co-cited references with the highest $K$ value in podocyte injury research.

\begin{tabular}{|c|c|c|c|c|c|}
\hline Cluster ID & Size & Silhouette & $\begin{array}{c}\text { Mean year } \\
\text { (Range) }\end{array}$ & Top term & $\begin{array}{l}\text { Log (likelihood } \\
\text { ratio) }\end{array}$ \\
\hline$\# 0$ & 92 & 0.827 & 2011 (2004-2018) & Cytoskeleton & 20.42 \\
\hline$\# 1$ & 86 & 0.873 & 2001 (1996-2007) & Diabetic nephropathy & 11.38 \\
\hline \#2 & 79 & 0.841 & 2001 (1994-2011) & Stretch & 14.03 \\
\hline \#3 & 77 & 0.847 & 2012 (2005-2020) & Autophagy & 59.85 \\
\hline \#4 & 74 & 0.866 & 2016 (2011-2020) & Diabetic kidney disease & 20.58 \\
\hline \#5 & 68 & 0.791 & 2007 (2001-2016) & Repair & 15.70 \\
\hline
\end{tabular}

Southern Medical University was the most cited author (cited 3,120 times), followed by Stuart J Shankland of the University of Washington (cited 2,730 times) and Matthias Kretzler of the University of Michigan (cited 2,613 times). The degree of cooperation among authors was relatively low and characterized by cooperation within the institution (Figure 3C).

\section{Core Journals}

All articles on podocyte injury were published in 413 journals. The top 10 journals with the highest productivity are shown in Table 4. About $31.32 \%$ of the articles were published in these journals. The Journal of the American Society of Nephrology (JASN) (published 159 articles, cited 10,305 times) was the most prolific journal, followed by Kidney International (KI) (published 148 articles, cited 8,895 times) and American Journal of Physiology-Renal Physiology (published 142 articles, cited 4,476 times). These three journals were also the most cited. The dual-map overlay shows three main citation paths. The published articles were mainly focused on journals in the field of molecular, biology, immunology and medicine, and medical clinical, whereas most of the cited articles were published in journals in the field of molecular, biology, genencs, health, nursing, and medicine (Figure 4).

\section{Co-Cited References}

The top 15 most cited references includes 7 reviews and 8 research articles (Table 5). Most articles focused on the pathophysiology and mechanism of podocyte injury. The most cited article was written by Susztak, who reported that a high-glucose environment can cause podocyte depletion, and leading to early DKD (cited 767 times) (Susztak et al., 2006). Subsequently, Inoki $\mathrm{K}$ et al. found that reducing the activity of mammalian target of rapamycin complex 1 in podocytes is a potential strategy for preventing DKD (cited 355 times) (Inoki et al., 2011), which further promoted the development of this field of study. We subsequently constructed a visualization network of cited references and performed a cluster analysis. A total of 14 clusters were found, the modularity $\mathrm{Q}$ was 0.6587 , and the mean silhouette value was 0.8617 (Supplementary Table S2). Six clusters with the highest K values were identified (Table 6; Figure 5A), which include "cytoskeleton," "diabetic nephropathy," and "stretch," among others. Furthermore, we performed a visualized timeline for clusters (Figure 5B). We found that "interstitial fibrosis" is an early field in podocyte injury. However, the current hotspots of podocyte injury are on "autophagy," "diabetic kidney disease," and "IncRNA." Finally, we conducted a reference burst. The top 25 references with the strongest citation bursts are shown in Supplementary Figure S2. Figure 5C shows the most representative references in terms of burst strength, burst duration, and burst time. We found that the works of Pavenstädt et al. (2003) have the highest bursts strength. In his article, he described the relationship between podocyte 

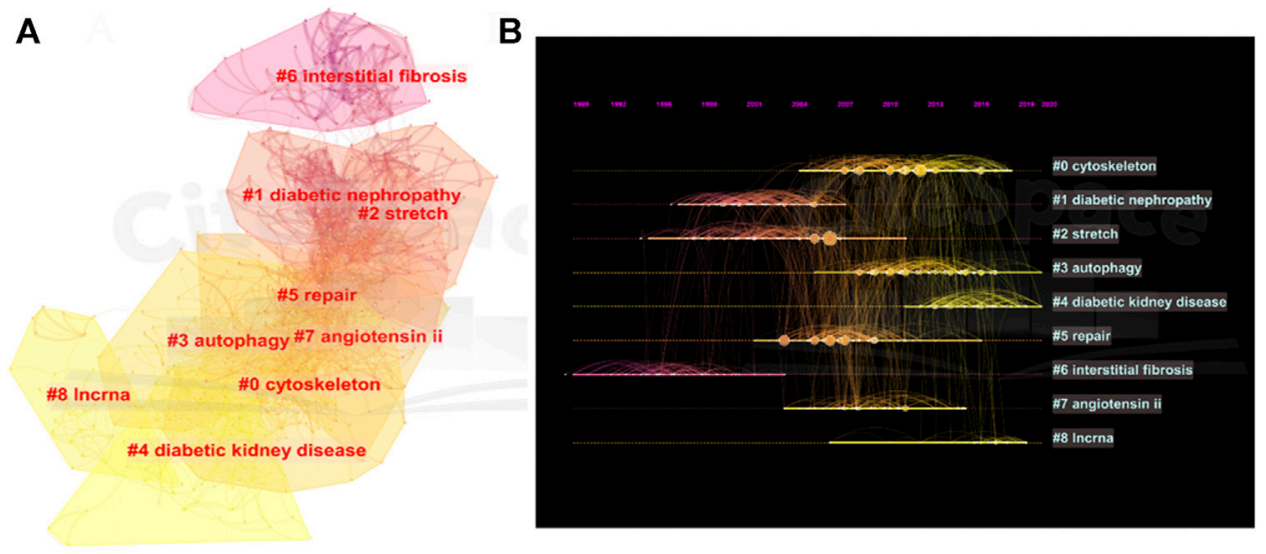

C

References

Barisoni L, 1999, J Am Soc Nephrol

Kaplan JM, 2000, Nat Genet

Boute N, 2000, Nat Genet

Schiffer M, 2001, J Clin Invest

Mundel P, 2002, J Am Soc Nephrol

Pavenstadt $\mathrm{H}_{3}$ 2003, Physiol Rev

Wolf G, 2005, Diabetes

Wharram BL, 2005, J Am Soc Nephrol

Winn MP, 2005, Science

Susztak K, 2006, Diabetes

Shankl and SJ, 2006, Kidney Int

Wiggins RC, 2007, Kidney Int

Nagata M, 2016, Kidney Int

Tagawa A, 2016, Diabetes

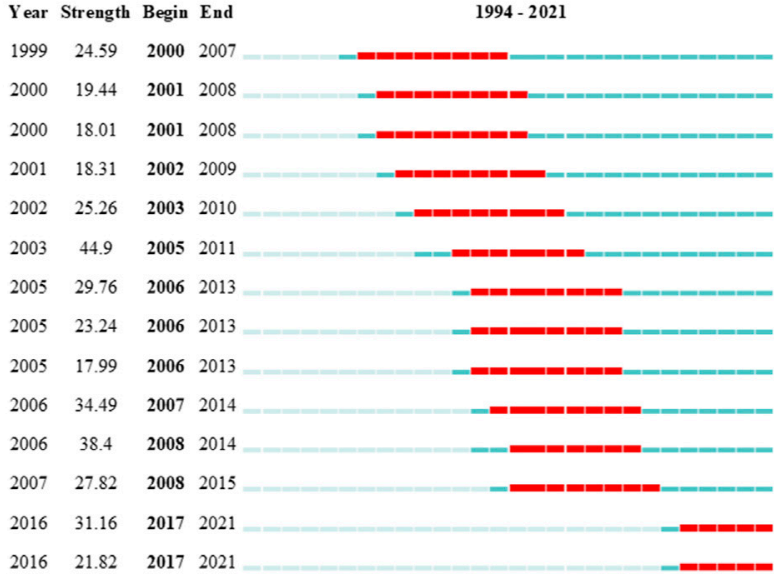

FIGURE 5 | Visualization of co-cited reference analysis. (A) Cluster Analysis of Co-cited References; (B) Timeline distribution of the top 9 clusters; (C) Representative burst references among top 25 references with the strongest citation bursts.

TABLE 7 | Keyword cluster analysis of podocyte injury research.

\begin{tabular}{|c|c|c|c|c|c|}
\hline Cluster ID & Size & Silhouette & Mean year & Top terms & $\begin{array}{l}\text { Log (likelihood } \\
\text { ratio) }\end{array}$ \\
\hline$\# 0$ & 66 & 0.551 & 2009 & Nephrotic syndrome & 128.11 \\
\hline$\# 1$ & 60 & 0.735 & 2003 & Proteinuria & 76.83 \\
\hline \#2 & 59 & 0.623 & 2011 & Diabetic nephropathy & 69.66 \\
\hline \#3 & 51 & 0.694 & 2009 & Oxidative stress & 53.16 \\
\hline$\# 4$ & 34 & 0.751 & 2011 & Autophagy & 50.62 \\
\hline \#5 & 31 & 0.61 & 2012 & Growth & 16.2 \\
\hline$\# 6$ & 31 & 0.734 & 2012 & Protein & 47.27 \\
\hline$\# 7$ & 22 & 0.779 & 2011 & Disease & 56.81 \\
\hline
\end{tabular}

injury and glomerulosclerosis Pavenstädt et al. (2003), indicating that this is the current focus in podocyte injury research. In addition, Nagata M's article has had a strong citation burst in recent years. His article introduced the correlation between autophagy and podocyte injury, indicating that this has garnered increased research interest in recent years (Nagata, 2016).

\section{Analysis of Keywords}

We extracted 4,080 keywords at data collection, and a cluster analysis revealed 8 clustering results (Table 7 ). The modularity $\mathrm{Q}$ of the cluster was 0.3087 , and the mean silhouette value was 0.7322 . The timeline of clustering showed that "diabetic nephropathy" and "proteinuria" were the most important areas of podocyte injury research, 


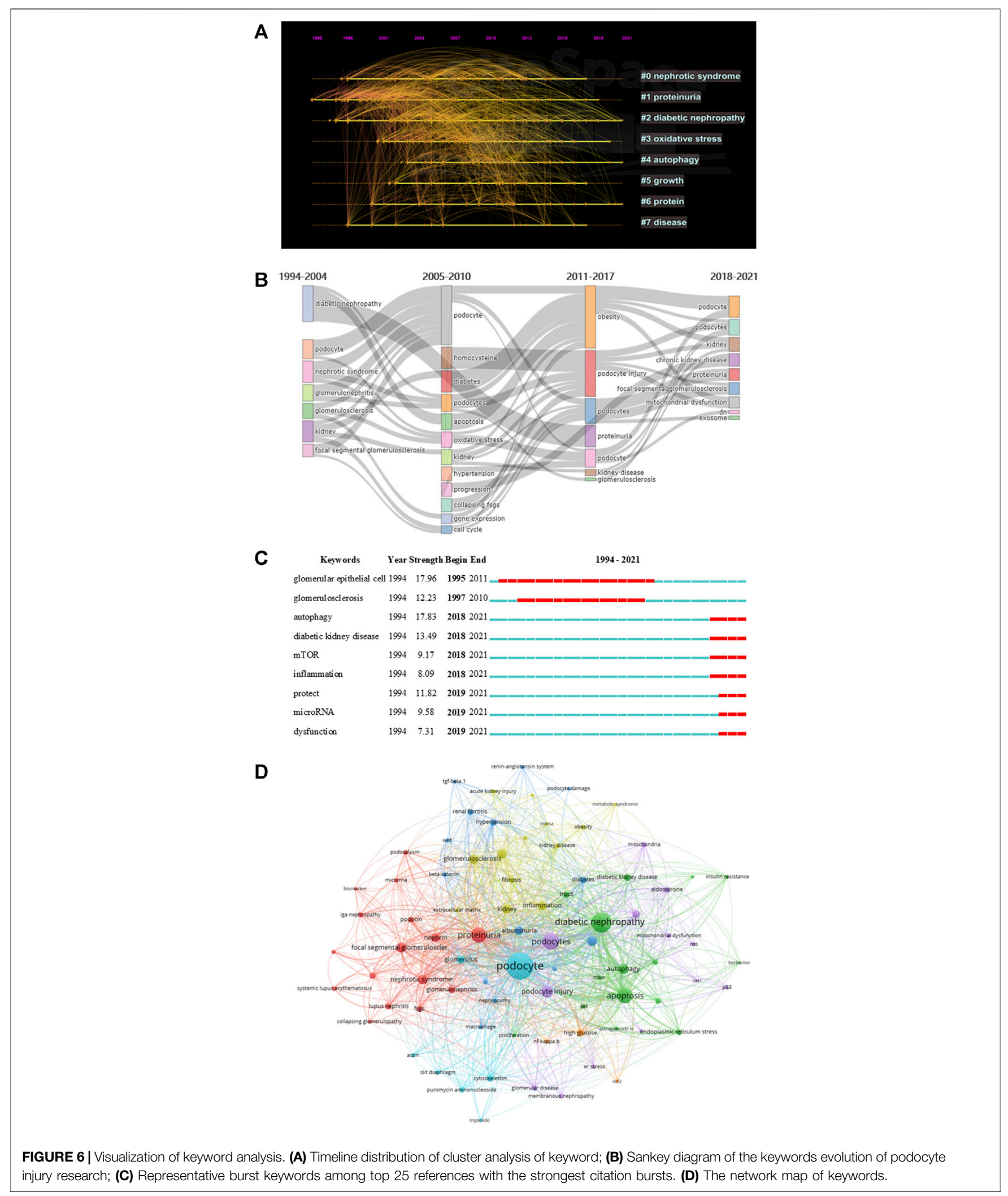




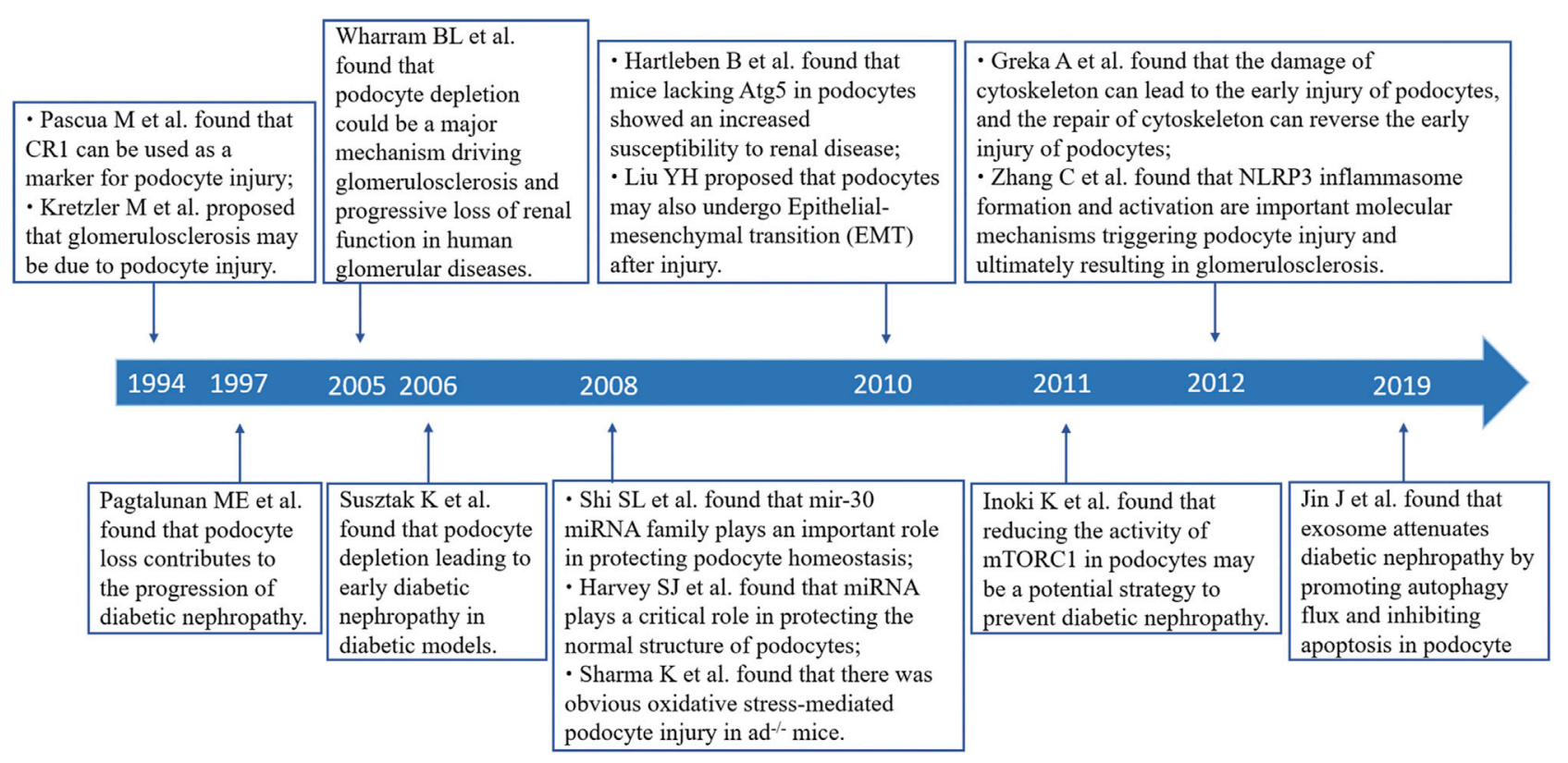

FIGURE 7 | Timeline of part of landmark achievements in podocyte injury research.

whereas "autophagy" was an emerging hotspot in podocyte injury studies (Figure 6A). We conducted a thematic evolution analysis on keywords, and found that the initial stages of research on podocyte injury was mainly focused on "diabetic nephropathy." However, with the maturity of the research field, the main research hotspot of podocyte injury has gradually evolved toward "homocysteine," "apoptosis," and "obesity," among others. In the past 3 years, "mitochondrial dysfunctions," "exosome," among others, and has gradually attracted the attention of scholars (Figure 6B). A total of 102 keywords were extracted by keyword burst analysis, the top 25 of which are shown in Supplementary Figure S3. We found that "autophagy" and "diabetic kidney disease" had the highest burst strength. In addition, we also found that "inflammation," "protect,", and "microRNA" were the latest keywords that emerged in the last 2 years (Figure 6C).

In addition, we followed the practice of (Kumar and Goel, 2021; Kumar et al., 2021) to explore the research gaps in the field of podocyte injury. We found that studies on podocyte injury were less exposed in acute kidney injury and $\operatorname{IgA}$ nephropathy, which may be related to the pathophysiological characteristics of podocytes. Notably, our study also found that the protective effect of traditional Chinese medicine monomer on podocyte injury is a promising blank field, and such as astragaloside iv (occurrences: 12), triptolide (occurrences: 10), berberine (occurrences: 7), etc. (Figure 6D). Xing et al. (2021) found that astragaloside iv can inhibit oxidative stress and alleviate podocyte damage. Coincidentally, Wan et al. (2021) found that triptolide can reverse the epithelial-mesenchymal transition of podocytes and improve podocyte-associated glomerular diseases. Therefore, the protective effect of traditional Chinese medicine monomer on podocyte injury needs more research to fill this gap.

\section{DISCUSSION}

In this study, we analyzed the main knowledge domain and emerging trends of podocyte injury using bibliometric analysis. Some landmark articles were also identified using this analysis (Figure 7). The results showed that the annual publications on podocyte injury generally show an upward trend and have entered a relatively mature research stage. The study of podocyte injury rose in 1994. Subsequently, Wharram et al. (2005) demonstrated that podocyte depletion may drive glomerulosclerosis and renal function progression in humans. The latest studies found that weight control was effective in preventing the kidney disease progression that occurs because podocytes fail to match the growth of the glomerular tuft (Fukuda et al., 2012).

China was the most productive country, and six of the top 10 productivity institutions are from China. Southern Medical University (published 68 articles, cited 1951 times) is the main representative, and Liu Youhua's team from this institution contributed most of the publications, and with a long-term research focus on $\mathrm{Wnt} / \beta$-catenin signaling and podocyte injury (Dai et al., 2009; He et al., 2011; Zhou et al., 2013). Their latest study found that the activation of the Wnt/ $\beta$-catenin signaling pathway mediates oxidative stress-induced podocyte injury (Zhou L. et al., 2019). The United States was the country with eight of the top 10 most cited articles. University of Washington (published 57 articles, cited 3,986 times) is the main 
representative. Shankland is a leader in the field of podocyte injury at this institution and has long devoted himself to the study of podocyte injury and glomerulosclerosis. His most cited article describes the relationship between podocyte injury and glomerulosclerosis (cited 589 times) (Shankland, 2006). His latest research found that Krüppel-like factor (KLF) was closely related to podocyte injury in glomerulonephritis. KLF15 expression contributes to the stability of cytoskeleton in podocyte (Mallipattu et al., 2017), whereas the loss of KLF4 activates the STAT3 signaling pathway, which further contributes to podocyte injury (Estrada et al., 2018).

Notably, among the top 10 core journals, JASN and KI had by far, the highest in both the number of articles published and the number of citations, and indicating that these two journals are the most popular journals for scholars who study podocyte injury. Scholars all over the world hope to publish landmark articles in JASN and KI. These two journals have published the most cutting-edge results and major breakthroughs in podocyte injury research. In recent years, Both JASN and KI have mainly focused on research exploring ways of protecting podocytes from damage. For example, a recent article published in KI found that interleukin 9 plays a protective role in the context of podocyte injury (Xiong et al., 2020). In the same period, an article published in JASN found that synaptopodin conferred a protective effect against podocyte injury. Differently, JASN focuses mainly on cutting-edge research, and whereas KI pays concentrates on breakthroughs in basic research.

The timeline view of references and keywords showed that studies related to podocyte injury are mostly basic studies, such as cytoskeleton, apoptosis, autophagy, repair, and interstitial fibrosis. An important review (cited 301 times) discusses the close relationship between cytoskeleton dysfunction and podocyte injury. Cytoskeleton damage can lead to the early injury of podocytes, and cytoskeleton repair can reverse early injury to podocytes (Greka and Mundel, 2012). Similarly, the study conducted by Faul C et al. found that cyclosporine A can prevent synaptopodin dephosphorylation and promote the stabilization of the podocyte cytoskeleton to reduce proteinuria (Faul et al., 2008). Coincidentally, Liu $M$ et al. recently found that sirt6 can maintain cytoskeletal stability and reduce podocyte apoptosis (Liu et al., 2017). Podocyte apoptosis, which is a special form of podocyte injury, exists in the early stages of DKD (Susztak et al., 2006). Schiffer M et al. found that podocyte apoptosis induced by transforming growth factor-beta existed in the early stage in FSGS mice (Schiffer et al., 2004). Oxidative stress has long been considered an important initiating factor of podocyte injury. In a highly cited article, Sharma $\mathrm{K}$ et al. found an obvious oxidative stress-mediated podocyte injury in adiponectin knockout (adiponectin ${ }^{-/-}$) mice (cited 464 times). Mo HY et al. found in their latest study that C-X-C chemokine receptor type 4 plays an important role in podocyte injury mediated by oxidative stress and can be used as a new target to improve podocyte injury (Mo et al., 2017).
Among the top 15 most cited articles, studies on DKD accounted for $46.67 \%(7 / 15)$, indicating that DKD is a hot focus of podocyte injury research. Pagtalunan ME et al. first found that podocyte loss was associated with DKD progression in Pima Indians with type II diabetes Pagtalunan et al. (1997). Subsequently, Nakamura $\mathrm{T}$ et al. found that the detection of podocytes in the urine was associated with the disease activity of DKD (Nakamura et al., 2000). At the same time, Coimbra TM et al. found that podocyte injury precedes glomerulosclerosis in diabetes mellitus (Coimbra et al., 2000) and that high glucoseand hypertension-related mechanical stress causes podocyte injury and proteinuria, which further progress to early DKD (Wolf et al., 2005). Isermann B et al. found that hyperglycemia caused the interruption of crosstalk between vascular compartment and podocytes, resulting in DKD (Isermann et al., 2007). Identifying new mediators of crosstalk between the vascular compartment and podocytes helps identify more effective treatments for DKD (Siddiqi and Advani, 2013).

With the development of podocyte damage research, some emerging research fields are gradually becoming the topics of interest of researchers. References and key bursts showed some items has had the highest bursts strength in the past 3 years. One of which is autophagy. Substantial evidence indicates that podocyte autophagy is a protective mechanism in kidney disease (Zeng et al., 2014). A landmark article on podocyte autophagy was published in 2010 by Hartleben B et al. who found that mice lacking autophagy-related 5 in podocytes showed an increased susceptibility to renal disease. Thus, they proposed that autophagy is a protective mechanism against glomerular injury (Hartleben et al., 2010). Lenoir O et al. found that hyperglycemia led to enhanced autophagic flux under the diabetic milieu, wherein autophagy in endothelial cells and podocytes inhibit the progression of DKD to glomerulosclerosis (Lenoir et al., 2015). A growing number of protein molecules that protect podocytes from injury by modulating autophagy have been found gradually, include Sirt6 (Liu et al., 2017), heme oxygenase-1 (Dong et al., 2015), and hepatocyte growth factor (Hou B. et al., 2020). Mitophagy has recently emerged as a new focus in podocyte injury research (Li et al., 2017; Zhou D. et al., 2019). Although an increasing number of studies on autophagy have been carried out, more in-depth research on autophagy are expected to be conducted in this field in the future. Another keyword with a significant citation burst is miRNA. Research on miRNA and podocyte injury has exploded because of the critical roles of miRNA. Many miRNA have protective effects on podocytes (Shi et al., 2008; Ishii et al., 2020), such as miRNA-21 (Kölling et al., 2017), miRNA-29 (Lin et al., 2014), and miRNA-30 (Wu et al., 2014). Strikingly, exosomal miRNA seems to be the specific focus in podocyte injury (Jin et al., 2019). Inflammation is the third hotspot in podocyte injury research. Inflammation is associated with podocyte dysfunction (Reidy et al., 2014). Many scholars have found that inflammasome activation leads to podocyte injury and glomerulosclerosis (Zhang et al., 2012; Abais et al., 2013; Shahzad et al., 2015). Notably, inflammation is an important 
bridge to podocyte injury studies. It often mediates podocyte injury together with autophagy and oxidative stress (Hou Y. et al., 2020; Dai et al., 2021).

Our research still has some limitations. First, this study only focused on podocyte injury, and we enriched the search strategy as much as possible. Still, some studies about podocytes were not included in the analysis. Second, most of the results of this study are based on machine algorithm, which is slightly insufficient in artificial induction. Finally, some new research fields related to podocyte injury may not have been included, and which may be due to the sensitivity of machine algorithms.

\section{CONCLUSION}

Using bibliometric analysis, we found that the research on podocyte injury has a good research prospect. Publications related to podocyte injury are increasing exponentially. DKD is the current focus of research in this field, and autophagy, microRNA, inflammasome, oxidative stress, and exosome are potential hotspots in podocyte injury research, which needs more focus. Notably, the protective effect of traditional Chinese medicine monomer on podocyte injury is less exposed, and more research is needed to fill this research gap.

\section{REFERENCES}

Abais, J. M., Zhang, C., Xia, M., Liu, Q., Gehr, T. W., Boini, K. M., et al. (2013). NADPH Oxidase-Mediated Triggering of Inflammasome Activation in Mouse Podocytes and Glomeruli during Hyperhomocysteinemia. Antioxid. Redox Signal. 18 (13), 1537-1548. doi:10.1089/ars.2012.4666

Alachkar, N., Wei, C., Arend, L. J., Jackson, A. M., Racusen, L. C., Fornoni, A., et al. (2013). Podocyte Effacement Closely Links to suPAR Levels at Time of Posttransplantation Focal Segmental Glomerulosclerosis Occurrence and Improves with Therapy. Transplantation 96 (7), 649-656. doi:10.1097/ TP.0b013e31829eda4f

Aria, M., and Cuccurullo, C. (2017). Bibliometrix : An R-Tool for Comprehensive Science Mapping Analysis. J. Informetrics 11 (4), 959-975. doi:10.1016/ j.joi.2017.08.007

Berger, K., Schulte, K., Boor, P., Kuppe, C., van Kuppevelt, T. H., Floege, J., et al. (2014). The Regenerative Potential of Parietal Epithelial Cells in Adult Mice. J. Am. Soc. Nephrol. 25 (4), 693-705. doi:10.1681/asn.2013050481

Chen, C. (2004). Searching for Intellectual Turning Points: Progressive Knowledge Domain Visualization. Proc. Natl. Acad. Sci. U S A. 101 (Suppl. 1), 5303-5310. doi:10.1073/pnas.0307513100

Chen, C., and Song, M. (2019). Visualizing a Field of Research: A Methodology of Systematic Scientometric Reviews. PLoS One 14 (10), e0223994. doi:10.1371/ journal.pone.0223994

Chen, C., and Leydesdorff, L. (2014). Patterns of Connections and Movements in Dual-Map Overlays: A New Method of Publication Portfolio Analysis. J. Assn Inf. Sci. Tec 65 (2), 334-351. doi:10.1002/asi.22968

Chen, C. (2017). Science Mapping: A Systematic Review of the Literature. J. Data Inf. Sci. 2 (2), 1-40. doi:10.1515/jdis-2017-0006

Coimbra, T. M., Janssen, U., Gröne, H. J., Ostendorf, T., Kunter, U., Schmidt, H., et al. (2000). Early Events Leading to Renal Injury in Obese Zucker (Fatty) Rats with Type II Diabetes. Kidney Int. 57 (1), 167-182. doi:10.1046/j.15231755.2000.00836.x

Dai, C., Stolz, D. B., Kiss, L. P., Monga, S. P., Holzman, L. B., and Liu, Y. (2009). Wnt/beta-catenin Signaling Promotes Podocyte Dysfunction and Albuminuria. J. Am. Soc. Nephrol. 20 (9), 1997-2008. doi:10.1681/ASN.2009010019

\section{DATA AVAILABILITY STATEMENT}

All datasets presented in this study can be found in the article and Supplementary Material.

\section{AUTHOR CONTRIBUTIONS}

TL and YZ designed the study. MY and YW collected the data. TL, LY, and HM analyzed the data and drafted the manuscript. All authors contributed to the article and approved the final version of the manuscript.

\section{FUNDING}

This work was supported by Grants from National Nature Science Foundation of China (82074393).

\section{SUPPLEMENTARY MATERIAL}

The Supplementary Material for this article can be found online at: https://www.frontiersin.org/articles/10.3389/fphar.2021.772386/ full\#supplementary-material

Dai, X., Liao, R., Liu, C., Liu, S., Huang, H., Liu, J., et al. (2021). Epigenetic Regulation of TXNIP-Mediated Oxidative Stress and NLRP3 Inflammasome Activation Contributes to SAHH Inhibition-Aggravated Diabetic Nephropathy. Redox Biol. 45, 102033. doi:10.1016/j.redox.2021.102033

Dong, C., Zheng, H., Huang, S., You, N., Xu, J., Ye, X., et al. (2015). Heme Oxygenase-1 Enhances Autophagy in Podocytes as a Protective Mechanism against High Glucose-Induced Apoptosis. Exp. Cel Res 337 (2), 146-159. doi:10.1016/j.yexcr.2015.04.005

Eng, D. G., Sunseri, M. W., Kaverina, N. V., Roeder, S. S., Pippin, J. W., and Shankland, S. J. (2015). Glomerular Parietal Epithelial Cells Contribute to Adult Podocyte Regeneration in Experimental Focal Segmental Glomerulosclerosis. Kidney Int. 88 (5), 999-1012. doi:10.1038/ki.2015.152

Estrada, C. C., Paladugu, P., Guo, Y., Pace, J., Revelo, M. P., Salant, D. J., et al. (2018). Krüppel-like Factor 4 Is a Negative Regulator of STAT3-Induced Glomerular Epithelial Cell Proliferation. JCI Insight 3 (12), e98214. doi:10.1172/jci.insight.98214

Faul, C., Donnelly, M., Merscher-Gomez, S., Chang, Y. H., Franz, S., Delfgaauw, J., et al. (2008). The Actin Cytoskeleton of Kidney Podocytes Is a Direct Target of the Antiproteinuric Effect of Cyclosporine A. Nat. Med. 14 (9), 931-938. doi:10.1038/nm.1857

Fukuda, A., Chowdhury, M. A., Venkatareddy, M. P., Wang, S. Q., Nishizono, R., Suzuki, T., et al. (2012). Growth-dependent Podocyte Failure Causes Glomerulosclerosis. J. Am. Soc. Nephrol. 23 (8), 1351-1363. doi:10.1681/ asn.2012030271

Garfield, E., Paris, S. W., and Stock, W. G. (2006). HistCite: A Software Tool for Informetric Analysis of Citation Linkage. NFD Information-Wissenschaft und Praxis 57 (8), 391-400.

Greka, A., and Mundel, P. (2012). Cell Biology and Pathology of Podocytes. Annu. Rev. Physiol. 74, 299-323. doi:10.1146/annurev-physiol-020911-153238

Hartleben, B., Gödel, M., Meyer-Schwesinger, C., Liu, S., Ulrich, T., Köbler, S., et al. (2010). Autophagy Influences Glomerular Disease Susceptibility and Maintains Podocyte Homeostasis in Aging Mice. J. Clin. Invest. 120 (4), 1084-1096. doi:10.1172/jci39492

He, W., Kang, Y. S., Dai, C., and Liu, Y. (2011). Blockade of Wnt/ $\beta$-Catenin Signaling by Paricalcitol Ameliorates Proteinuria and Kidney Injury. J. Am. Soc. Nephrol. 22 (1), 90-103. doi:10.1681/asn.2009121236 
Hou, B., Li, Y., Li, X., Zhang, C., Zhao, Z., Chen, Q., et al. (2020a). HGF Protected against Diabetic Nephropathy via Autophagy-Lysosome Pathway in Podocyte by Modulating PI3K/Akt-Gsk3 $\beta$-TFEB axis. Cell Signal 75, 109744. doi:10.1016/j.cellsig.2020.109744

Hou, Y., Lin, S., Qiu, J., Sun, W., Dong, M., Xiang, Y., et al. (2020b). NLRP3 Inflammasome Negatively Regulates Podocyte Autophagy in Diabetic Nephropathy. Biochem. Biophys. Res. Commun. 521 (3), 791-798. doi:10.1016/j.bbrc.2019.10.194

Inoki, K., Mori, H., Wang, J., Wang, J., Suzuki, T., Suzuki, T., et al. (2011). mTORC1 Activation in Podocytes Is a Critical Step in the Development of Diabetic Nephropathy in Mice. J. Clin. Invest. 121 (6), 2181-2196. doi:10.1172/ JCI44771

Isermann, B., Vinnikov, I. A., Madhusudhan, T., Herzog, S., Kashif, M., Blautzik, J., et al. (2007). Activated Protein C Protects against Diabetic Nephropathy by Inhibiting Endothelial and Podocyte Apoptosis. Nat. Med. 13 (11), 1349-1358. doi:10.1038/nm1667

Ishii, H., Kaneko, S., Yanai, K., Aomatsu, A., Hirai, K., Ookawara, S., et al. (2020). MicroRNAs in Podocyte Injury in Diabetic Nephropathy. Front. Genet. 11, 993. doi:10.3389/fgene.2020.00993

Jin, J., Shi, Y., Gong, J., Zhao, L., Li, Y., He, Q., et al. (2019). Exosome Secreted from Adipose-Derived Stem Cells Attenuates Diabetic Nephropathy by Promoting Autophagy Flux and Inhibiting Apoptosis in Podocyte. Stem Cel Res Ther 10 (1), 95. doi:10.1186/s13287-019-1177-1

Kölling, M., Kaucsar, T., Schauerte, C., Hübner, A., Dettling, A., Park, J.-K., et al. (2017). Therapeutic miR-21 Silencing Ameliorates Diabetic Kidney Disease in Mice. Mol. Ther. 25 (1), 165-180. doi:10.1016/j.ymthe.2016.08.001

Kretzler, M., Koeppen-Hagemann, I., and Kriz, W. (1994). Podocyte Damage Is a Critical Step in the Development of Glomerulosclerosis in the Uninephrectomised-Desoxycorticosterone Hypertensive Rat. Virchows Arch. 425 (2), 181-193. doi:10.1007/bf00230355

Kumar, R., and Goel, P. (2021). Exploring the Domain of Interpretive Structural Modelling (ISM) for Sustainable Future Panorama: A Bibliometric and Content Analysis. Arch. Computat Methods Eng. doi:10.1007/s11831-021-09675-7

Kumar, R., Singh, S., Sidhu, A. S., and Pruncu, C. I. (2021). Bibliometric Analysis of Specific Energy Consumption (SEC) in Machining Operations: A Sustainable Response. Sustainability 13 (10), 5617. doi:10.3390/su13105617

Lenoir, O., Jasiek, M., Hénique, C., Guyonnet, L., Hartleben, B., Bork, T., et al. (2015). Endothelial Cell and Podocyte Autophagy Synergistically Protect from Diabetes-Induced Glomerulosclerosis. Autophagy 11 (7), 1130-1145. doi:10.1080/15548627.2015.1049799

Li, W., Du, M., Wang, Q., Ma, X., Wu, L., Guo, F., et al. (2017). FoxO1 Promotes Mitophagy in the Podocytes of Diabetic Male Mice via the PINK1/Parkin Pathway. Endocrinology 158 (7), 2155-2167. doi:10.1210/en.2016-1970

Lin, C. L., Lee, P. H., Hsu, Y. C., Lei, C. C., Ko, J. Y., Chuang, P. C., et al. (2014). MicroRNA-29a Promotion of Nephrin Acetylation Ameliorates Hyperglycemia-Induced Podocyte Dysfunction. J. Am. Soc. Nephrol. 25 (8), 1698-1709. doi:10.1681/asn.2013050527

Liu, M., Liang, K., Zhen, J., Zhou, M., Wang, X., Wang, Z., et al. (2017). Sirt6 Deficiency Exacerbates Podocyte Injury and Proteinuria through Targeting Notch Signaling. Nat. Commun. 8 (1), 413. doi:10.1080/ 15384101.2020.178348110.1038/s41467-017-00498-4

Makino, S. I., Shirata, N., Oliva Trejo, J. A., Yamamoto-Nonaka, K., Yamada, H., Miyake, T., et al. (2021). Impairment of Proteasome Function in Podocytes Leads to CKD. J. Am. Soc. Nephrol. 32 (3), 597-613. doi:10.1681/ asn. 2019101025

Mallipattu, S. K., Guo, Y., Revelo, M. P., Roa-Peña, L., Miller, T., Ling, J., et al. (2017). Krüppel-Like Factor 15 Mediates Glucocorticoid-Induced Restoration of Podocyte Differentiation Markers. J. Am. Soc. Nephrol. 28 (1), 166-184. doi:10.1681/asn.2015060672

Mo, H., Wu, Q., Miao, J., Luo, C., Hong, X., Wang, Y., et al. (2017). C-X-C Chemokine Receptor Type 4 Plays a Crucial Role in Mediating Oxidative Stress-Induced Podocyte Injury. Antioxid. Redox Signal. 27 (6), 345-362. doi:10.1089/ars.2016.6758

Nagata, M. (2016). Podocyte Injury and its Consequences. Kidney Int. 89 (6), 1221-1230. doi:10.1016/j.kint.2016.01.012

Nakamura, T., Ushiyama, C., Suzuki, S., Hara, M., Shimada, N., Ebihara, I., et al. (2000). Urinary Excretion of Podocytes in Patients with Diabetic Nephropathy. Nephrol. Dial. Transpl. 15 (9), 1379-1383. doi:10.1093/ndt/15.9.1379
Pagtalunan, M. E., Miller, P. L., Jumping-Eagle, S., Nelson, R. G., Myers, B. D., Rennke, H. G., et al. (1997). Podocyte Loss and Progressive Glomerular Injury in Type II Diabetes. J. Clin. Invest. 99 (2), 342-348. doi:10.1172/ jci119163

Pascual, M., Steiger, G., Sadallah, S., Paccaud, J. P., Carpentier, J. L., James, R., et al. (1994). Identification of Membrane-Bound CR1 (CD35) in Human Urine: Evidence for its Release by Glomerular Podocytes. J. Exp. Med. 179 (3), 889-899. doi:10.1084/jem.179.3.889

Pavenstädt, H., Kriz, W., and Kretzler, M. (2003). Cell Biology of the Glomerular Podocyte. Physiol. Rev. 83 (1), 253-307. doi:10.1152/physrev.00020.2002

Reidy, K., Kang, H. M., Hostetter, T., and Susztak, K. (2014). Molecular Mechanisms of Diabetic Kidney Disease. J. Clin. Invest. 124 (6), 2333-2340. doi:10.1172/jci72271

Schell, C., and Huber, T. B. (2017). The Evolving Complexity of the Podocyte Cytoskeleton. J. Am. Soc. Nephrol. 28 (11), 3166-3174. doi:10.1681/ asn. 2017020143

Schiffer, M., Mundel, P., Shaw, A. S., and Böttinger, E. P. (2004). A Novel Role for the Adaptor Molecule CD2-Associated Protein in Transforming Growth Factor-Beta-Induced Apoptosis. J. Biol. Chem. 279 (35), 37004-37012. doi:10.1074/jbc.M403534200

Shahzad, K., Bock, F., Dong, W., Wang, H., Kopf, S., Kohli, S., et al. (2015). Nlrp3inflammasome Activation in Non-myeloid-derived Cells Aggravates Diabetic Nephropathy. Kidney Int. 87 (1), 74-84. doi:10.1038/ki.2014.271

Shankland, S. J. (2006). The Podocyte's Response to Injury: Role in Proteinuria and Glomerulosclerosis. Kidney Int. 69 (12), 2131-2147. doi:10.1038/sj.ki.5000410

Shi, S., Yu, L., Chiu, C., Sun, Y., Chen, J., Khitrov, G., et al. (2008). Podocyteselective Deletion of Dicer Induces Proteinuria and Glomerulosclerosis. J. Am. Soc. Nephrol. 19 (11), 2159-2169. doi:10.1681/asn.2008030312

Siddiqi, F. S., and Advani, A. (2013). Endothelial-podocyte Crosstalk: the Missing Link between Endothelial Dysfunction and Albuminuria in Diabetes. Diabetes 62 (11), 3647-3655. doi:10.2337/db13-0795

Susztak, K., Raff, A. C., Schiffer, M., and Böttinger, E. P. (2006). Glucose-induced Reactive Oxygen Species Cause Apoptosis of Podocytes and Podocyte Depletion at the Onset of Diabetic Nephropathy. Diabetes 55 (1), 225-233. doi:10.2337/diabetes.55.01.06.db05-0894

van Eck, N. I., and Waltman, L. (2010). Software Survey: VOSviewer, a Computer Program for Bibliometric Mapping. Scientometrics 84 (2), 523-538. doi:10.1007/s11192-009-0146-3

Wan, F., Tang, Y. W., Tang, X. L., Li, Y. Y., and Yang, R. C. (2021). TET2 Mediated Demethylation Is Involved in the Protective Effect of Triptolide on Podocytes. Am. J. Transl Res. 13 (3), 1233-1244.

Wang, S., Zhou, H., Zheng, L., Zhu, W., Zhu, L., Feng, D., et al. (2021). Global Trends in Research of Macrophages Associated with Acute Lung Injury over Past 10 years: A Bibliometric Analysis. Front. Immunol. 12, 669539. doi:10.3389/fimmu.2021.669539

Wharram, B. L., Goyal, M., Wiggins, J. E., Sanden, S. K., Hussain, S., Filipiak, W. E., et al. (2005). Podocyte Depletion Causes Glomerulosclerosis: Diphtheria ToxinInduced Podocyte Depletion in Rats Expressing Human Diphtheria Toxin Receptor Transgene. J. Am. Soc. Nephrol. 16 (10), 2941-2952. doi:10.1681/ asn. 2005010055

Wolf, G., Chen, S., and Ziyadeh, F. N. (2005). From the Periphery of the Glomerular Capillary wall toward the center of Disease: Podocyte Injury Comes of Age in Diabetic Nephropathy. Diabetes 54 (6), 1626-1634. doi:10.2337/diabetes.54.6.1626

Wu, J., Zheng, C., Fan, Y., Zeng, C., Chen, Z., Qin, W., et al. (2014). Downregulation of microRNA-30 Facilitates Podocyte Injury and Is Prevented by Glucocorticoids. J. Am. Soc. Nephrol. 25 (1), 92-104. doi:10.1681/asn.2012111101

Xing, L., Fang, J., Zhu, B., Wang, L., Chen, J., Wang, Y., et al. (2021). Astragaloside IV Protects against Podocyte Apoptosis by Inhibiting Oxidative Stress via Activating PPAR $\gamma$-Klotho-FoxO1 axis in Diabetic Nephropathy. Life Sci. 269, 119068. doi:10.1016/j.lfs.2021.119068

Xiong, T., Attar, M., Gnirck, A. C., Wunderlich, M., Becker, M., Rickassel, C., et al. (2020). Interleukin-9 Protects from Early Podocyte Injury and Progressive Glomerulosclerosis in Adriamycin-Induced Nephropathy. Kidney Int. 98 (3), 615-629. doi:10.1016/j.kint.2020.04.036

Zeng, C., Fan, Y., Wu, J., Shi, S., Chen, Z., Zhong, Y., et al. (2014). Podocyte Autophagic Activity Plays a Protective Role in Renal Injury and Delays the 
Progression of Podocytopathies. J. Pathol. 234 (2), 203-213. doi:10.1002/ path.4382

Zhang, C., Boini, K. M., Xia, M., Abais, J. M., Li, X., Liu, Q., et al. (2012). Activation of Nod-like Receptor Protein 3 Inflammasomes Turns on Podocyte Injury and Glomerular Sclerosis in Hyperhomocysteinemia. Hypertension 60 (1), 154-162. doi:10.1161/hypertensionaha.111.189688

Zhou, D., Zhou, M., Wang, Z., Fu, Y., Jia, M., Wang, X., et al. (2019a). PGRN Acts as a Novel Regulator of Mitochondrial Homeostasis by Facilitating Mitophagy and Mitochondrial Biogenesis to Prevent Podocyte Injury in Diabetic Nephropathy. Cell Death Dis 10 (7), 524. doi:10.1038/s41419-0191754-3

Zhou, L., Chen, X., Lu, M., Wu, Q., Yuan, Q., Hu, C., et al. (2019b). Wnt/ $\beta$-catenin Links Oxidative Stress to Podocyte Injury and Proteinuria. Kidney Int. 95 (4), 830-845. doi:10.1016/j.kint.2018.10.032

Zhou, L., Li, Y., Zhou, D., Zhou, D., Tan, R. J., and Liu, Y. (2013). Loss of Klotho Contributes to Kidney Injury by Derepression of Wnt/ $\beta$-Catenin Signaling. J. Am. Soc. Nephrol. 24 (5), 771-785. doi:10.1681/ASN.2012080865
Conflict of Interest: The authors declare that the research was conducted in the absence of any commercial or financial relationships that could be construed as a potential conflict of interest.

Publisher's Note: All claims expressed in this article are solely those of the authors and do not necessarily represent those of their affiliated organizations, or those of the publisher, the editors and the reviewers. Any product that may be evaluated in this article, or claim that may be made by its manufacturer, is not guaranteed or endorsed by the publisher.

Copyright (0) 2021 Liu, Yang, Mao, Ma, Wang and Zhan. This is an open-access article distributed under the terms of the Creative Commons Attribution License (CC $B Y$ ). The use, distribution or reproduction in other forums is permitted, provided the original author(s) and the copyright owner(s) are credited and that the original publication in this journal is cited, in accordance with accepted academic practice. No use, distribution or reproduction is permitted which does not comply with these terms. 